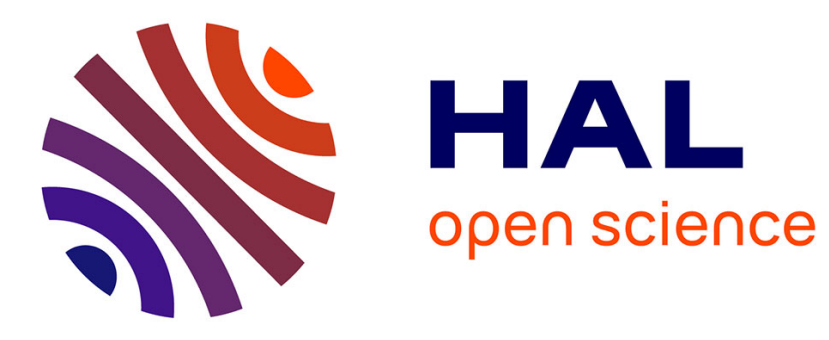

\title{
Mobility State Estimation in LTE
}

Majed Haddad, Dalia-Georgiana Herculea, Eitan Altman, Nidham Ben

Rached, Véronique Capdevielle, Chung Shue Chen, Frédéric Ratovelomanana

\section{To cite this version:}

Majed Haddad, Dalia-Georgiana Herculea, Eitan Altman, Nidham Ben Rached, Véronique Capdevielle, et al.. Mobility State Estimation in LTE. IEEE Wireless Communications and Networking Conference, IEEE, Apr 2016, Doha, Qatar. 10.1109/WCNC.2016.7564917 . hal-01291728

\section{HAL Id: hal-01291728 \\ https://hal.inria.fr/hal-01291728}

Submitted on 27 May 2016

HAL is a multi-disciplinary open access archive for the deposit and dissemination of scientific research documents, whether they are published or not. The documents may come from teaching and research institutions in France or abroad, or from public or private research centers.
L'archive ouverte pluridisciplinaire HAL, est destinée au dépôt et à la diffusion de documents scientifiques de niveau recherche, publiés ou non, émanant des établissements d'enseignement et de recherche français ou étrangers, des laboratoires publics ou privés. 


\section{Mobility State Estimation in LTE}

\author{
Majed Haddad \\ University of Avignon \\ Avignon, France
}

\author{
Dalia Georgiana Herculea \\ Bell Labs \\ Villarceaux, France
}

\author{
Eitan Altman \\ INRIA Sophia-Antipolis \\ Sophia-Antipolis, France
}
Nidham Ben Rached
Nokia
Villarceaux, France

\author{
Véronique Capdevielle \\ Nokia \\ Villarceaux, France
}

\author{
Chung Shue Chen \\ Bell Labs \\ Villarceaux, France
}

\author{
Frédéric Ratovelomanana \\ Nokia \\ Villarceaux, France
}

\begin{abstract}
Estimating mobile user speed is a problematic issue which has significant impacts to radio resource management and also to the mobility management of Long Term Evolution (LTE) networks. This paper introduces two algorithms that can estimate the speed of mobile user equipments (UE), with low computational requirement, and without modification of neither current user equipment nor 3GPP standard protocol. The proposed methods rely on uplink (UL) sounding reference signal (SRS) power measurements performed at the eNodeB (eNB) and remain efficient with large sampling period (e.g., 40 ms or beyond). We evaluate the effectiveness of our algorithms using realistic LTE system data provided by the eNB Layer1 team of Alcatel-Lucent. Results show that the classification of UE's speed required by LTE can be achieved with high accuracy. In addition, they have minimal impact to the central processing unit (CPU) and the memory of eNB modem. We see that they are very practical to today's LTE networks and would allow a continuous and real-time UE speed estimation.
\end{abstract}

Index Terms-LTE, mobility state estimation, speed estimation, heterogeneous networks.

\section{INTRODUCTION}

The large penetration of mobile devices with wireless connectivity provides new capabilities to support various smart applications and services. Due to their small size and popularity, mobile devices allow users to access wireless networks anywhere and anytime while undergoing various activities and all kinds of mobility, raising new challenges to wireless systems and protocols. This creates regimes where wireless networks are pushed to operate close to their performance limits, dictated by current architectural considerations. Besides, to enhance network capacity for increasing traffic demands [1], cellular networks become heterogeneous networks (HetNets) by adding small cells (e.g., metro, pico or femtocells either indoor or outdoor) to existing macro cells.

With the deployment of HetNets, mobility management has become a more challenging issue because of the increased

These results have been produced in the framework of the common research lab between INRIA and Bell labs.

This work has been done when Majed Haddad was with INRIA SophiaAntipolis, France. complexity due to inter-layer Radio Resource Management (RRM), between macro and small cells. A mobile user has a great freedom to switch from one cell to another, whereas the network must ensure continuous service and high-quality user experience. Supporting service continuity is especially challenging due to potentially high handover frequency and new requirement of ultra low latency [2]. However, if the UE's mobility information is available for mobility management and network optimization, the network can proactively adapt to the user and guarantee seamless and smooth UE handover with optimal cell selection to ensure good end-user quality and high performance [3].

Knowledge of UE mobility can also be very beneficial to transmission scheduling [4], mobility load balancing [5], channel quality indicator (CQI) feedback enhancement [6], energy efficiency enhancement [7], and many resource management scenarios. For example, choosing the most suitable channel dependent scheduling scheme for LTE, either frequency selective scheduling (FSS) or frequency diversity scheduling (FDS), would depend on the user velocity. Frequency selective scheduling shall be preferred for low-speed UEs. At high UE speed, due to significant Doppler effect, the frequency dependent channel information is not accurate such that it would be better to employ FDS.

To address the issue of mobility, 3GPP Release 9 introduces mobility robustness optimization (MRO) features to the self-optimization functions [8]. The objective of MRO is to dynamically improve the network performance of handovers (HO) in order to provide enhanced high Quality of Experience (QoE) for the users as well as increased network capacity. This is done by automatically adapting cell parameters to adjust handover thresholds based on feedback of performance indicators.

\section{A. Contributions}

In this paper, we address the problem of determining the speed or speed class of the UE for mobility management in LTE radio access network (RAN). We present two different methods for UE speed estimation, which rely on already available SRS power measurements performed by LTE base station, called eNB. The solutions aim at taking advantage 
of the speed dependent time variations of slow fading (i.e., shadowing) in the SRS measurements. These properties are captured by a metric defined by each method. The metric is computed online and then compared with a reference curve or a look-up table (database), with respect to the shadowing decorrelation distance $D$ (see Fig. 1). The first method, Spectral Analysis Method (SAM), evaluates the maximum frequency of oscillation of SRS measurements, built on Fast Fourier Transform (FFT). The second one, Time-based Spectrum Spreading Method (TSSM), evaluates in time domain the speed dependent spectrum spreading of the SRS signal. From a system deployment standpoint, TSSM is easier to implement as SAM requires an accurate selection of the frequency peak, which may be difficult to obtain under some conditions. In terms of speed estimation accuracy, both TSSM and SAM have good performance. Note that the computational cost of TSSM is very low. It has very limited impact to the CPU of the base station (eNB). Both methods can be deployed in current networks and can allow a continuous and real-time UE speed estimation (a.k.a. speed tracking).

Note that the proposed methods belong to network-based solutions according to 3GPP-LTE standard since they are based on UL SRS power measurements conducted at the eNB (the eNB measures the SRS transmitted by UE). However, notice that this is not a restriction of the proposed algorithms, which may also be implemented at the mobile UE depending on system or implementation preference. In that case, we may rather use LTE downlink Reference Signal Received Power (RSRP) instead of SRS. Besides, the methods we propose allow large sampling period, which implies that UEs can send SRS less frequently and save energy. Clearly, the more frequently the UE sends SRS, the higher will be the speed estimation accuracy. However, this would come at the cost of higher energy consumption. For the UE power saving purpose (there is always a hard energy constraint), we resort to explore the characteristics of large-scale fading, namely the shadow fading, and use it to estimate UE speed, under large sampling period. It is known that the channel variation due to shadowing would become significant when the receiver moves over distances greater than several tens of the carrier wavelength. Besides, in practice, SRS fast fading often would be flattened during measurements when performing power averaging in LTE. For these reasons, we devise solutions by using the large-scale shadow fading characteristics but not the small-scale fast fading (see existing methods in Section I-B). In addition, we target computationally efficient methods, with minimal impact to the CPU and memory of eNB modem.

\section{B. Existing Speed Estimation Techniques}

Several methods were proposed to estimate the speed of UEs in some specific use cases. Crossing based methods count the number of times that a process crosses a certain level [9]. Covariance based methods estimate the speed by computing the covariance function of the received signal [10], [11]. Maximum likelihood (ML) based methods rely on periodic channel estimation [12]. Power spectrum based methods estimate the
Doppler frequency based on the characteristics of the power spectrum of the fading channel. The main drawback of these methods is that they do not cope with the large periodicity of LTE SRS measurements since they assume analyzing the speed dependent fast fading characteristics of the signals. In practice, due to large sampling period of SRS, the Nyquist frequency for avoiding spectrum aliasing is reduced, which limits the maximum observable Doppler frequency and thus decreases the maximum observable UE speed. Furthermore, it is known that crossing methods are less efficient than covariance methods for small observation windows. Both techniques are sensitive to noise for small Doppler spreads. The ML based methods could provide near-optimal performance but require the knowledge of the signal-to-noise-ratio (SNR) and the Gaussian noise level and have high implementation complexity. Another approach based on timing advancement (TA) updates has been recently developed. However, this method only provides estimate of radial speed and thus under estimates actual speed, which may lead to steer high speed UEs to small cells resulting in an increase in $\mathrm{HO}$ failures and call drop rate.

\section{SySTEM MODEL}

A wireless channel can be modeled by a series of random variables to represent their impact on the strength of the signal received at the UE side. In principle, the channel link gain can be decoupled into the following components: the distancedependent path loss, large-scale slow channel variation which is often caused by large scale (shadow) fading, and small scale (fast) variation.

As aforementioned, in this work we explore channel fading characteristics for estimating user speed. The following statistical model has been developed for land mobile radio systems see for example [13] in which the amplitude of the channel $r(t)$ is a product of the envelope of a Rayleigh process, which is denoted by $\beta(t)$, and a shadowing process $\psi(t)$ such that

$$
r(t)=\beta(t) \cdot \psi(t) .
$$

Note that the Rayleigh distributed process is to account for fast fading and is given by the envelope of a complex Gaussian random process. On the other hand, the shadowing can be modeled by a log-normal random variable. Using Gudmundson's correlation model which is also adopted by 3GPP standard [14], we can write $\psi(t)$ as a first-order auto-regressive process in the spatial domain by a lognormal process:

$$
\psi(t)=e^{\frac{\sigma_{\psi_{d B}} \psi_{d B}(t)+\mu_{\psi_{d B}}}{20}}
$$

where the parameters $\sigma_{\psi_{d B}}$ and $\mu_{\psi_{d B}}$ are called the shadow standard deviation and mean respectively. Note that both of them are in decibel $(\mathrm{dB})$ values. Meanwhile, $\psi(t)$ is a spatial Gaussian process and has the following lognormal probability density function (PDF):

$$
p\left(\psi_{d B}\right)=\frac{1}{\sqrt{2 \pi} \sigma_{\psi_{d B}}} e^{-\frac{\left(\psi_{d B}-\mu_{\psi_{d B}}\right)^{2}}{2 \sigma_{\psi_{d B}}{ }^{2}}} .
$$




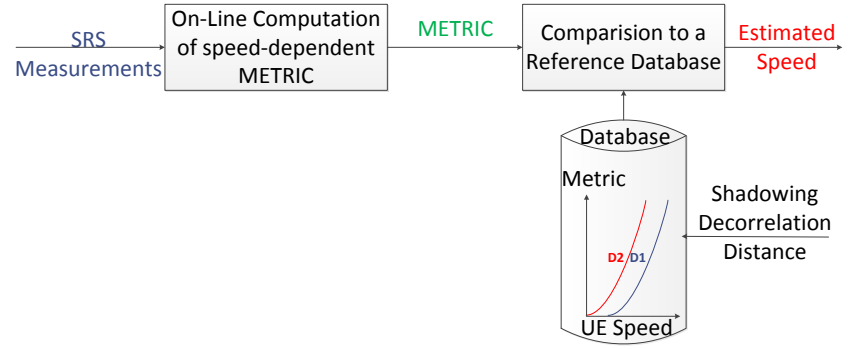

Fig. 1: The functional elements of the proposed solution.

The spatial autocorrelation between the shadow fadings at two points separated by a distance $\delta$ is known as:

$$
\begin{aligned}
\mathcal{R}_{\psi}(\delta) & =\mathbb{E}\left[\left(\psi(d-\delta)-\mu_{\psi_{d B}}\right)\left(\psi(d)-\mu_{\psi_{d B}}\right)\right] \\
& =\sigma_{\psi_{d B}}{ }^{2} \rho^{\frac{\delta}{D}}
\end{aligned}
$$

where $\rho$ is the correlation between two points separated by a certain distance $D$, and $d$ is the dummy variable. For example, measurements indicate that for sub-urban macro cells with a frequency carrier $f_{c}=900 \mathrm{MHz}, \rho=0.82$ for $D=100 \mathrm{~m}$, whereas for urban micro cells with $f_{c} \simeq 2 \mathrm{GHz}, \rho=0.3$ for $D=10 \mathrm{~m}$ (see [15]-[17]). This model can be simplified after removing its empirical dependence by letting $\rho=1 / e$, which yields

$$
\mathcal{R}_{\psi}(\delta)=\sigma_{\psi_{d B}}^{2} e^{-\delta / D}
$$

where $D$ is called the decorrelation distance. It refers to how far does a mobile has to move for the shadowing to change. This decorrelation distance usually varies with the propagation environment and carrier frequency and should be obtained empirically.

When the receiver is mobile, the spatial autocorrelation can be translated into time autocorrelation such that the shadowing behaves as a correlated time-varying process. The time autocorrelation can be obtained from (5) by substituting $\delta$ by $v \tau$ such that

$$
\begin{aligned}
\mathcal{R}_{\psi}(\tau) & =\mathbb{E}\left[\left(\psi(t-\tau)-\mu_{\psi}\right)\left(\psi(t)-\mu_{\psi}\right)\right] \\
& =\sigma_{\psi}^{2} e^{-\frac{v \tau}{D}}
\end{aligned}
$$

where $v$ is the UE's speed and $t$ is the time variable.

The central idea is to define a metric that is an implicit function of the autocorrelation function of SRS measurements. This metric is related to $v / D$, since the autocorrelation is a function of $v / D$ as shown in (6). This speed dependency of the metric will enable us to distinguish UE speeds, by comparison to a reference database (i.e., a look-up table) that provides oneto-one mapping between the metric and UE speed, for a given decorrelation distance $D$.

As shown in Fig. 1, there are three functional elements in our solutions:

1) Online computation of method-specific metric for currently received SRS measurements.
2) Database is set up offline which includes reference data and is represented by a curve, function or look-up table (LUT).

3) Comparison of the above metric (computed online) with the reference curve or look-up table. The UE speed is thus deduced, in considering the speed corresponding to the computed metric, which is obtained during 1).

\section{Spectral Analysis Method (SAM)}

The measured signal strengths are subject to speed dependent variations which can be explored through a spectral analysis for revealing specific information such as the frequency, phase and amplitude of the component sinusoids. With low speed UE, the SRS measurements vary slowly since they are often highly correlated for a long duration, whereas with high speed UE, the period of high correlation is relatively short. Our spectral representation of the SRS measurements aims to capture this speed dependent "oscillation" frequency of the shadow fading. For the spectral analysis of SRS, we compute the power spectrum density (PSD), i.e., the Fourier transform of the autocorrelation function, as follows:

$$
\mathcal{S}_{\psi}(f)=\int_{0}^{\infty} \mathcal{R}_{\psi \psi}(\tau) e^{-j 2 \pi f \tau} d \tau
$$

By replacing $\mathcal{R}_{\psi \psi}(\tau)$ with its expression in (6), we obtain after some calculations:

$$
\mathcal{S}_{\psi}(f)=\frac{\sigma_{\psi}^{2}}{\pi} \frac{f_{0}}{f^{2}-f_{0}^{2}}
$$

which is a Lorentzian function with $f_{0}=\frac{v}{D}$. Therefore a "resonance" frequency appears under the effect of velocity at frequency $f=\frac{v}{D}$. This resonance frequency is used as our SAM metric.

The spectral analysis method (SAM) consists in detecting the speed dependent maximum oscillation frequency of shadow fading (i.e., the resonance frequency). This is obtained by detecting the peak frequency after fast Fourier transform (FTT) of SRS measurements. This peak frequency is then compared to a reference database (see Fig. 1) which provides a one-to-one mapping between the peak frequency at the database and the one computed at the UE side. The database is built based on measurements that are collected in the field and processed offline with FFT. In Fig. 2, we show ${ }^{1}$ the SAM metric under different decorrelation distances for a block period size of $16 \mathrm{~s}$. We notably compare the theoretical peak frequency expressed as

$$
f_{\text {peak }}=\frac{v}{2 T D}
$$

and the simulated peak frequency. $T$ in the expression above stands for the measurements sampling period. One can observe that the theoretical peak frequency provides a very good match to the peak frequency determined from simulation results. The peak frequency increases linearly with $v$. For a fixed speed

\footnotetext{
${ }^{1}$ The holes in the curves are only due to lack of data in the intervals in question.
} 


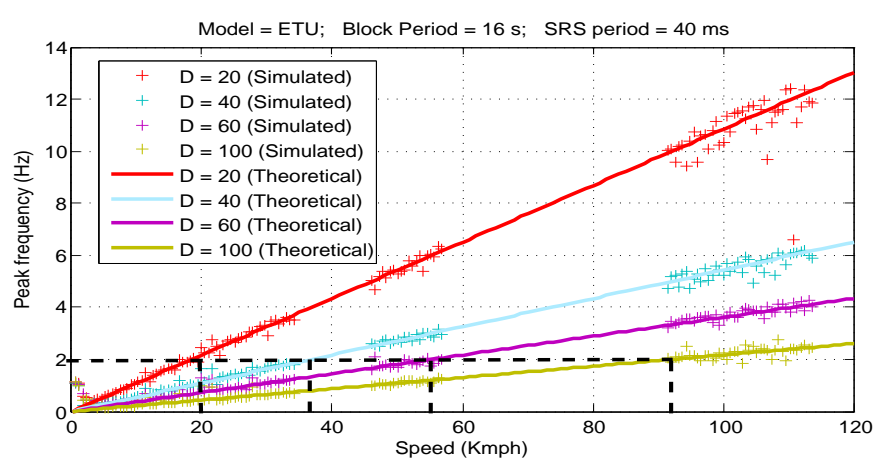

Fig. 2: Simulated vs. theoretical (Eq. 9) SAM metric as function of the decorrelation distance $D$ in meters.

$v$, it can be verified from Fig. 2 that the peak frequency is a monotonic decreasing function of $D$. Moreover, it is clear that the error on $v$ will increase due to the error on $D$ since we have from (9) that

$$
v=2 T D f_{\text {peak }} .
$$

SAM requires the calculation of one FFT per speed estimation which induces a non negligible computational cost and requires large memory, especially for large size of processed blocks: $256 \cdot \ln (256)=1419$ operations per UE speed estimation for blocks of 256 samples and $512 \cdot \ln (512)=3194$ operations per UE speed estimation for blocks of 512 samples.

\section{Time-based Spectral Spreading Method} (TSSM)

Considering zero mean of the received signal amplitude $r(t)$, we obtain after deriving the autocorrelation function of the received signal with respect to $\tau$

$$
\begin{aligned}
\frac{\partial \mathcal{R}_{r r}(\tau)}{\partial \tau} & =\mathbb{E}\left[r(t) \frac{\partial r(t+\tau)}{\partial \tau}\right] \\
& =-\mathbb{E}[r(t) \dot{r}(t+\tau)] \\
& =\mathcal{R}_{r \dot{r}}(\tau) .
\end{aligned}
$$

Under the assumption of stationarity of the autocorrelation function $\mathcal{R}_{r r}$, when deriving again the above equation, one gets

$$
\frac{\partial^{2} \mathcal{R}_{r r}(\tau)}{\partial^{2} \tau}=-\frac{\partial}{\partial \tau} \mathbb{E}[r(t+\tau) \dot{r}(t)]
$$

which gives from [18]

$$
\begin{aligned}
\frac{\partial^{2} \mathcal{R}_{r r}(\tau)}{\partial^{2} \tau} & =-\mathbb{E}\left[\frac{\partial r(t+\tau)}{\partial \tau} \dot{r}(t)\right] \\
& =-\mathbb{E}[\dot{r}(t+\tau) \dot{r}(t)] .
\end{aligned}
$$

In particular, the absolute value of the above second derivation of $\mathcal{R}_{r r}$ when $\tau$ tends to $0^{+}$is as follows:

$$
\lim _{\tau \rightarrow 0^{+}}\left(\left|\frac{\partial^{2} \mathcal{R}_{r r}(\tau)}{\partial^{2} \tau}\right|\right)=\mathbb{E}\left[(\dot{r}(t))^{2}\right]
$$

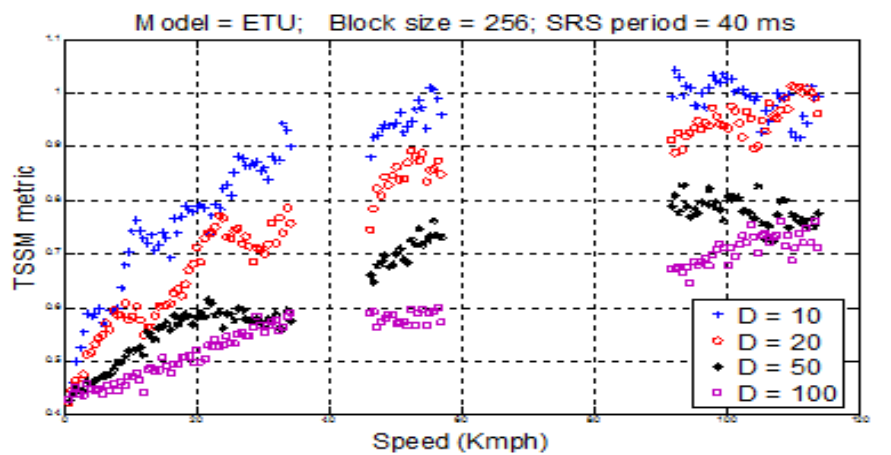

Fig. 3: TSSM metric as function of the decorrelation distance $D$ in meters.

Taking the value of the second derivative at $\tau=0$, we have

$$
{\frac{\partial^{2} \mathcal{R}_{r r}(\tau)}{\partial^{2} \tau}}_{\tau=0}=\mathcal{R}_{\dot{r} \dot{r}}(0)=\mathbb{E}\left[(\dot{r}(t))^{2}\right] .
$$

Using the expression of the autocorrelation function of the shadowing $\mathcal{R}_{\psi}(\tau)$ in (6), the second derivative of the autocorrelation function of the shadowing at the left hand side of (13) can be expressed as

$$
{\frac{\partial^{2} \mathcal{R}_{\psi \psi}(\tau)}{\partial^{2} \tau}}_{\tau=0}=\frac{\sigma_{\psi}^{2} v^{2}}{D^{2}} .
$$

We can see that the second derivative of the autocorrelation of the shadowing is proportional to the square of the speed. Combining (15) and (16), we find a relation between the speed $v$ and the variance of the derivative of the SRS measurements $r(t)$, namely

$$
\mathbb{E}\left[(\dot{r}(t))^{2}\right] \sim \frac{\sigma_{\psi}^{2} v^{2}}{D^{2}} .
$$

The TSSM metric is deduced from Eq. (17). It is obtained from SRS measurements by computing

$$
\sqrt{\mathbb{E}\left[\left(\dot{r}_{N}(t)\right)^{2}\right]} \sim \frac{v}{D}
$$

where $r_{N}$ is the normalized SRS signal, i.e., $r_{N}(t)=\frac{r(t)}{\sigma_{\psi}}$.

Remark 1. We note that the above TSSM metric equals to $v / D$ corresponding to $f_{\text {peak }}=v / D$, which is the spectrum spreading of the power spectral density in (8) in the time domain. Fig. 3 depicts the TSSM metric computed according to Eq. (18) as function of the speed. As expected, we find a similar result to SAM: the TSSM metric is a linearly increasing function of the speed $v$, which increases for decreasing $D$. This is due to the fact that the two metrics are inversely proportional to $D$ as pointed out by Eq. (9) for SAM and Eq. (18) for TSSM.

We end up by having the following TSSM speed estimate approximation as a function of the normalized received signal amplitude $r_{N}(t)$, namely 


$$
v \sim D \sqrt{\mathbb{E}\left[\left(\dot{r}_{N}(t)\right)^{2}\right]} .
$$

TSSM aims to detect the speed dependent spreading of the power spectral density in the time domain, by exploiting the variance of the temporal derivative of the SRS signal, after normalization. A TSSM metric is then determined and compared to a reference database for determining the UE speed class. The reference database is embedded in the software of the eNB and provides a one-to-one mapping between TSSM metric and UE speed. It is built based on measurements from the field or experimental work offline. The main functional elements of TSSM are the normalization operation, the computation of derivatives and the computation of variance. The method has a much lower computation complexity than for SAM. It requires around 10 operations per UE speed estimation and a buffer size of 256 samples. In what follows, we detail the implementation steps required for TSSM as described in Fig. 4.

Taking blocks of $N$ samples of SRS measurements, denoted by $X_{i}=\left[X_{i+1} \ldots X_{i+N}\right]$, the signal is first normalized:

$$
X_{k}=\frac{X_{k}}{\sqrt{\text { norm }\left(X_{i}\right)}} .
$$

Next, the derivatives are computed over the temporal blocks as follows:

$$
d_{k}=\frac{\mathbb{E}\left[X_{k}\right]-\mathbb{E}\left[X_{(k-n)}\right]}{n T}
$$

where $n$ is the delay between two derivative points. This operation enables to capture only the slow variations of shadowing through the smoothing of the signal and therefore reducing fast fading and Doppler variations. The TSSM metric is then computed per block $i$ from the set of all derivatives $d_{k}$ below

$$
T S S M_{i}=\sqrt{\left.\frac{1}{K} \sum_{k=1}^{K}\left(d_{k}-m\right)^{2}\right)}
$$

where $K$ is the number of derivatives computed on block $i$ and $m$ is the mean value over these $K$ derivatives. Finally, the estimated metric in (22) is compared to the reference database. The speed that corresponds to the metric in the database that is closest to the metric we computed, is outputted as the estimated UE speed.

\section{Simulations Results}

We evaluate the two proposed methods in 3GPP-LTE setup by using realistic eNB power measurements of UL SRS provided by the eNB Layer1 team of Alcatel-Lucent. The signals are instantaneous wideband SRS with $10 \mathrm{MHz}$ bandwidth, 1.9 $\mathrm{GHz}$ carrier frequency, a time sampling periods $T$ of $40 \mathrm{~ms}$ and a Doppler frequency varying from 1 to $166 \mathrm{~Hz}$ (low, medium and high Doppler frequencies). The radio channel follows the 3GPP Extended Typical Urban (ETU) environment requirement. We consider the speed classification performance with 4 UE speed classes: The first 3 classes are $[0,30),[30,60)$ and $[60,90) \mathrm{kmph}$. The last class is related to UE speed

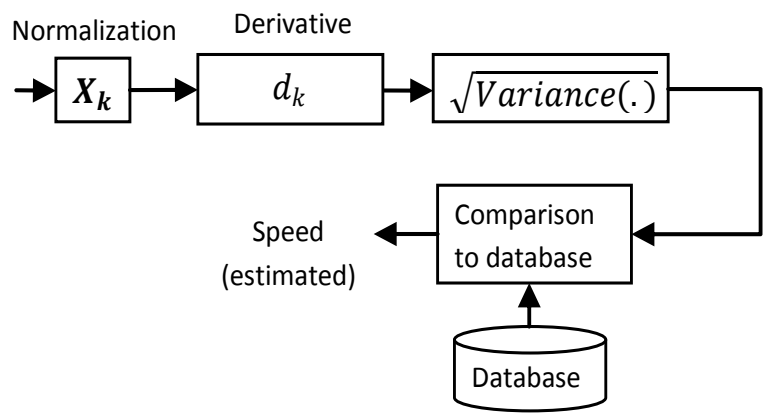

Fig. 4: TSSM algorithm.

\begin{tabular}{|c|c|c|}
\hline Decorrelation distance & $D=20 \mathrm{~m}$ & $D=50 \mathrm{~m}$ \\
\hline Number of FFT samples $=256$ & $(7,15)$ & $(14,15)$ \\
\hline Number of FFT samples $=512$ & $(1,7)$ & $(8,7)$ \\
\hline
\end{tabular}

TABLE I: (Bias, Standard deviation) in kmph of speed error estimation for SAM.

greater than or equal to $90 \mathrm{kmph}$. We define the following performance metrics:

- Let $P_{0}(D)$ be the probability of correct speed classification,

- Let $P_{1}(D)$ be the probability of classifying the UE speed into the right class or the neighbor (immediately higher or lower) speed class.

While computing the probability of speed classification, we further consider errors on the shadowing decorrelation distance $D$. The term "no error on $D$ " stands for the case where the algorithm has chosen the right mapping (among the possible $D$ s) for the UE metric computed online and the metric computed offline in the database. An error of $x \%$ means that the algorithm has chosen a wrong mapping with an error of $x \%$ on the right $D$.

\section{A. Spectral Analysis Method (SAM)}

We report in Table I the bias and the standard deviation of the error on the speed estimates using SAM method, considering 256 and 512 FFT samples. The simulations are done for a decorrelation distance of $20 \mathrm{~m}$ and $50 \mathrm{~m}$. The performance is slightly degraded with larger $D$ (in particular the bias, and is improved by an increase on the FFT block size (from 256 samples to 512 samples) as, with higher FFT samples, more shadowing oscillation periods can be observed.

Table II depicts the probability of correct classification with 4 speed classes. As expected, we first notice that the probability of correct speed class estimation is improved with the increase in the number of samples, since a larger number of samples allows to better capture the variation of the shadowing effect. We also observe that the probability of correct classification is always greater than or equal to $95 \%$ if the decorrelation distance mapping is correctly selected. 


\begin{tabular}{|l|c|c|c|c|}
\hline & $P_{0}(D)[\%]$ & $P_{0}(D)[\%]$ & $P_{1}(D)[\%]$ & $P_{1}(D)[\%]$ \\
\hline $\begin{array}{l}\text { Number of } \\
\text { FFT samples }\end{array}$ & 256 & 512 & 256 & 512 \\
\hline $\begin{array}{l}\text { No error on } \\
D\end{array}$ & $P_{0}(20)=95$ & $P_{0}(20)=99$ & $P_{1}(20)=99$ & $P_{1}(20)=99$ \\
$P_{0}(50)=95$ & $P_{0}(50)=97$ & $P_{1}(50)=99$ & $P_{1}(50)=99$ \\
\hline $\begin{array}{l}\text { With an error } \\
\text { of 50\% on } D\end{array}$ & $P_{0}(20)=54$ & $P_{0}(20)=60$ & $P_{1}(20)=97$ & $P_{1}(20)=99$ \\
$P_{0}(50)=52$ & $P_{0}(50)=59$ & $P_{1}(50)=97$ & $P_{1}(50)=99$ \\
\hline
\end{tabular}

TABLE II: Probability of speed classification with 4 UE speed classes for SAM.

\begin{tabular}{|c|c|c|}
\hline Decorrelation distance & $D=20 \mathrm{~m}$ & $D=50 \mathrm{~m}$ \\
\hline Block size $=256$ & $(12,12)$ & $(12,12)$ \\
\hline Block size $=512$ & $(7,6)$ & $(7,6)$ \\
\hline
\end{tabular}

TABLE III: (Bias, Standard deviation) in $\mathrm{kmph}$ of speed error estimation for TSSM.

\section{B. Time-based Spectral Spreading Method (TSSM)}

Table III reports the average bias and the standard deviation of the speed estimation errors using TSSM method, considering block size of 256 or 512 samples, and decorrelation distances of 20 and 50 meters. Here, the bias and standard deviation are around 12 meters for both $D=20$ $\mathrm{m}$ and $50 \mathrm{~m}$. They decrease to 7 and $6 \mathrm{~m}$ respectively, by increasing the block size (from 256 to 512 samples). Table IV summarizes speed classification probabilities with 256 samples considering 4 UE speed classes. The probability of correct speed classification with 4 classes is always higher than $98 \%$ when the database is correctly selected.

Remark 2. It is noteworthy, that the bias values in Tables I and III has not been taken into account in the speed estimation procedure. The classification probabilities in Table II for SAM, and in Table IV for TSSM will be naturally further enhanced after bias subtraction.

\section{CONCLUSION AND PERSPECTIVES}

We have addressed the problem of estimating the UE speed in LTE radio access network by means of network-based algorithms. The two methods presented here rely on SRS measurements performed by the eNB with a large periodicity (40 ms or more). While most of the existing speed estimation methods exploit fast fading characteristics, the proposed methods make use of the properties of slow fading (shadowing). These properties are captured either through a spectral analysis method (for SAM), or through the analysis in time domain of the speed dependent power spectrum spreading (for TSSM) of the received signal. In addition to the fact that they do not require any modification from a UE standpoint, both SAM and TSSM have shown good performances in terms of probability of successful speed classification (with

\begin{tabular}{|l|l|l|}
\hline & $P_{0}(D)[\%]$ & $P_{1}(D)[\%]$ \\
\hline No error on $D$ & $P_{0}(20)=98$ & $P_{1}(20)=99$ \\
& $P_{0}(50)=98$ & $P_{1}(50)=99$ \\
\hline With an error of $50 \%$ on $D$ & $P_{0}(20)=64$ & $P_{1}(20)=80$ \\
& $P_{0}(50)=62$ & $P_{1}(50)=74$ \\
\hline
\end{tabular}

TABLE IV: Probability of speed classification with 4 UE speed classes and 256 samples for TSSM.

$80 \%$ minimum), provided that the lookup table in the data base is properly selected. TSSM has a lower computational requirement and CPU memory than SAM, enabling UE speed tracking through adaptive implementation.

\section{REFERENCES}

[1] A. Damnjanovic, J. Montojo, Y. Wei, T. Ji, T. Luo, M. Vajapeyam, T. Yoo, O. Song, and D. Malladi, "A survey on 3GPP heterogeneous networks," IEEE Wireless Communications, vol. 18, no. 3, pp. 10-21, 2011.

[2] Samsung, "Mobility support to pico cells in the co-channel HetNet deployment," in 3GPP Standard Contribution (R2-104017), Mar. 2010.

[3] Z. Lu and G. de Veciana, "Optimizing stored video delivery for mobile networks: The value of knowing the future," in INFOCOM, 2013 Proceedings IEEE, April 2013, pp. 2706-2714.

[4] J. Niu, D. Lee, T. Su, G. Li, and X. Ren, "User classification and scheduling in lte downlink systems sith heterogeneous user mobilities," IEEE Transactions on Wireless Communications, vol. 12, no. 12, pp. 6205-6213, December 2013.

[5] S. M. Musa and N. F. Mir, "An analytical approach for mobility load balancing in wireless networks." CIT, vol. 19, no. 3, pp. 169-176, 2011

[6] A. Dua, F. Lu, and V. Sethuraman, "Speed-adaptive channel quality indicator (cqi) estimation," Jun. 28 2012, uS Patent App. 13/253,039. [Online]. Available: http://www.google.com/patents/US20120163207

[7] A. Prasad, P. Lunden, O. Tirkkonen, and C. Wijting, "Energy-efficient flexible inter-frequency scanning mechanism for enhanced small cell discovery," in Vehicular Technology Conference (VTC Spring), June 2013, pp. 1-5.

[8] 3GPP TR 36.902 V9.3.1 (2011-03), "Evolved universal terrestrial radio access (e-utra); mobility enhancements in heterogeneous networks," in Tech. Rep. V11.1.0, Jan. 2013.

[9] H. Zhang and A. Abdi, "Mobile speed estimation using diversity combining in fading channels," in IEEE GLOBECOM, Nov 2004.

[10] J. Holtzman and A. Sampath, "Adaptive averaging methodology for handoffs in cellular systems," Vehicular Technology, IEEE Transactions on, vol. 44, no. 1, pp. 59-66, Feb 1995.

[11] Y. Zheng and C. Xiao, "Mobile speed estimation for broadband wireless communications over rician fading channels," Wireless Communications, IEEE Transactions on, vol. 8, no. 1, pp. 1-5, Jan 2009.

[12] H. Arslan, L. Krasny, D. Koilpillai, and S. Chennakeshu, "Doppler spread estimation for wireless mobile radio systems," in IEEE WCNC, 2000.

[13] H. Suzuki, "A statistical model for urban radio propogation," IEEE Transactions on Communications, vol. 25, no. 7, pp. 673-680, Jul 1977.

[14] 3GPP TR 36.814 V2.0.0 (2010-3), “3rd generation partnership project; technical specification group radio access network; further advancements for e-utra physical layer aspects (release 9)," in Tech. Rep.

[15] M. Marsan and G. Hess, "Shadow variability in an urban land mobile radio environment," Electronics Letters, vol. 26, p. 646648, May 1990.

[16] A. Algans, K. Pedersen, and P. Mogensen, "Experimental analysis of the joint statistical properties of azimuth spread, delay spread, and shadow fading," Selected Areas in Communications, IEEE Journal on, vol. 20, no. 3, pp. 523-531, Apr 2002.

[17] J. Weitzen and T. Lowe, "Measurement of angular and distance correlation properties of log-normal shadowing at $1900 \mathrm{mhz}$ and its application to design of pcs systems," Vehicular Technology, vol. 51, no. 2, pp. 265273, Mar 2002.

[18] J. S. Bendat and A. G. Piersol, Random Data: Analysis and Measurement Procedures. Wiley Series in Probability and Statistics, Mar. 2010. 